\title{
PADRÃO DE ACOMETIMENTO DA COLUNA CERVICAL NA ARTRITE REUMATOIDE: SEGUIMENTO DE 6 ANOS
}

\author{
PATTERN OF INVOLVEMENT OF THE CERVICAL COLUMN IN RHEUMATOID ARTHRITIS:
} A 6 YEAR FOLLOW UP

\section{CUADRO DE AFECTACIÓN DE LA COLUMNA CERVICAL EN LA ARTRITIS REUMATOIDEA: SEGUIMIENTO DE 6 AÑOS}

Aurélio Felipe Arantes ${ }^{1}$, André luiz Passos Cardoso², Murilo Tavares Daher ${ }^{3}$, Newton Antônio Tristão4 ${ }^{4}$, Wilson Eloy Pimenta Júnior ${ }^{4}$, Nilzio ANTONIO dA SILVAF ${ }^{5}$ SÉrgio DAHER ${ }^{6}$.

\begin{abstract}
RESUMO
Objetivos: Foi avaliado o padrão de acometimento cervical em pacientes com diagnóstico de artrite reumatoide, correlacionando-se os achados de imagem com quadro neurológico, medicação utilizada, tempo de evolução da doença, idade de início da doença, exames laboratoriais e quadro clínico. Métodos: Esse mesmo grupo de pacientes tinha sido estudado há 6 anos, e esses dados foram comparados para avaliar se houve piora do padrão de acometimento ao longo do tempo. Foram feitas radiografias simples da coluna cervical, nas incidências ântero-posterior, perfil neutro e dinâmico. A classificação de Ranawat foi utilizada para avaliar a sintomatologia dolorosa e o envolvimento neurológico. Foram utilizados os testes de regressão logística univariada $(p<0,05)$. Resultados: Foram avaliados 24 dos 35 pacientes que compuseram a amostra anterior, havendo perda de seguimento de 7. Encontramos algumas correlações estatisticamente significativas: reflexo bicciptal $(p=0,049)$ e método de Ranawat ( $p=0,023$, no raio-x em extensão e $p=0,034$, no raio-x neutro) com coluna estável, erosão do dente do áxis $(p=0,048)$ e subluxação lateral $(p=0,022)$ com instabilidade atlanto-axial e sinal de Lhermithé $(p=0,025)$ com invaginação basilar. Instabilidade cervical foi encontrada em 66,6\% (16/24), havendo um aumento estatisticamente significante da instabilidade subaxial nos últimos 6 anos $(p=0,032)$. Conclusões: Em ordem decrescente, as instabilidades foram: subluxação atlanto-axial com 50\%, subluxação subaxial com 41,6\% e invaginação basilar com 16,6\%. Houve um aumento no número de pacientes com instabilidade subaxial, não se observando correlação com a faixa etária de acometimento. Também não se observou correlação do reflexo biciptal com qualquer tipo de instabilidade e sim com a coluna cervical estável, resultado diferente do encontrado anteriormente.
\end{abstract}

Descritores: Artrite Reumatoide; Doenças Ósseas; Raios X; Testes Hematológicos; Sinais e Sintomas.

\begin{abstract}
Objective: The long-term pattern of cervical involvement in patients with a diagnosis of rheumatoid arthritis was investigated, correlating the imaging findings with neurological symptoms, medication used, duration of the disease, age of onset, laboratory tests and clinical picture. Methods: This same group of patients had already been studied for some time, and data were compared to verify if there was a worsening of the involvement pattern throughout time. Plain X-rays were obtained from the cervical column, in anteroposterior and neutral and dynamic lateral views. The Ranawat classification was used to evaluate pain symptoms and neurological involvement. For the analysis, the authors used the univariate logistic regression tests $(p<0.05)$. Results: Twenty-four of the 35 patients from the previous sample were evaluated and seven were lost to follow up. The authors found some statistically significant correlations: bicipital reflex $(p=0.049)$ and the Ranawat method ( $p=0.023$ with extension X-ray and $p=0.034$ with a neutral $X$-ray) with a stable column, erosion of the dens axis ( $p=0.048)$ and lateral subluxation $(p=0.22)$ with atlanto-axial instability and the Lhermitte signal $(p=0.025)$ with basilar invagination. Cervical instability was found in 66.6\% (16/24) of the patients, and there was a significant statistical increase of the subaxial instability for the past 6 years $(p=0.032)$. Conclusions: In a decreasing order of occurrence, the authors were able to verify the following types of instability: atlanto-axial subluxation, 50\%; subaxial subluxation, 41.6\%; and basilar invagination, 16.6\%. An increase in the number of patients with subaxial instability was verified, and no correlation was observed regarding the age of onset. Additionally, no correlation was found between the bicipital reflex and any type of instability but rather with a stable column, and this result is different from what the authors obtained in the previous series.
\end{abstract}

Keywords: Rheumatoid Arthritis, Bone Diseases; X-ray; Blood tests; Signs and Symptoms.

\section{RESUMEN}

Objetivos: Fue evaluado el cuadrode afectación cervical en pacientes con diagnóstico de artritis reumatoidea, correlacionando los hallazgos de imagen con cuadro neurológico, medicación utilizada, tiempo de evolución de la enfermedad, edad del paciente alinicio de la enfermedad, exámenes de laboratorio y cuadro clínico. Métodos: Ese mismo grupo de pacientes había sido estudiado 6 años antes, y esos datos recolectados fueron comparados para evaluar si hubo un empeoramiento del cuadrode afectacióna lo largo del tiempo. Se hicieron radiografías simples de la columna cervical, en las incidencias antero posterior, perfil neutro y dinámico. La clasificación de Ranawat fue utilizada para evaluar la sintomatología dolorosa y la involucración neurológica. Se utilizaron las pruebas de regresión logística univariada

\footnotetext{
1. Estagiário do Serviço de Cirurgia da Coluna do Departamento de Ortopedia eTraumatologia da Faculdade de Medicina da UFG, Goiânia.GO.

2. Médico Assistente do Serviço de Cirurgia da Coluna do Departamento de Ortopedia eTraumatologia da Faculdade de Medicina da UFG, Goiânia.GO, e Mestre em Ciências da Saúde pela UnB.

3. Professor do Departamento de Ortopedia eTraumatologia da Faculdade de Medicina da UFG.

4. Médico Assistente do Serviço de Cirurgia da Coluna do Departamento de Ortopedia eTraumatologia da Faculdade de Medicina da UFG, Goiânia.GO.

5. Professor Titular de Reumatologia do Departamento de Clinica Médica da Faculdade de Medicina da UFG, Goiânia.GO, Doutor em Reumatologia pela FMUSP

6. Professor e Chefe do Serviço de Cirurgia da Coluna Vertebral do Departamento de Ortopedia da Faculdade de Medicina da UFG, Goiânia.GO.
}

Trabalho realizado no Hospital das Clínicas da Universidade Federal de Goiás. Brasil.

Correspondência: Rua T-64, № 751, Quadra 154, Lote 15, Ed. Rio das Ostras, Setor Bueno, Goiânia-GO. Brasil.. Email: aureliofelipe@gmail.com 
$(p<0,05)$. Resultados: Evaluados 24 de los 35 pacientes que formaron parte de la muestra anterior, habiendo pérdida de seguimiento de 7. Encontramos algunas correlaciones estadísticamente significativas: reflejo bicipital $(p=0,049)$ y método de Ranawat $(p=0,023$, en el Rayo $X$ en extensión a $p=0,034$, en el Rayo X neutro) con columna estable, erosión del odontoide $(p=0,048)$ y subluxación lateral $(p=0,022)$ con inestabilidad atlanto-axial y señal de Lhermithé $(p=0,025)$ con invaginación basilar. Se ha encontrado inestabilidad cervical en un 66,6\% (16/24), habiendo un aumento significativamente estadístico de la inestabilidad subaxial en los últimos 6 años p=0,032). Conclusiones: En orden decreciente, las inestabilidades han sido estas: subluxación atlantoaxial con un 50\%, subluxación subaxial, con un 41,6\% e invaginación basilar, con un 16,6\%. Hubo un aumento en el número de pacientes con inestabilidad subaxial, aunque no se ha observado correlación de ese tipo de inestabilidad con el nivel de edad de afectación. Tampoco se ha observado correlación del reflejo bicipital con cualquier tipo de inestabilidad, sino con la columna cervical estable, resultado distinto del que había sido encontrado en la serie anterior.

Descriptores: Artritis reumatoidea; Enfermedades Óseas; Rayo X; Pruebas Hematológicas; Señales y Síntomas.

\section{INTRODUÇÃO}

A artrite reumatoide (AR) é uma doença inflamatória crônica sistêmica, caracterizada pela progressiva poliartrite com subsequente destruição articular, geralmente simétrica ${ }^{1}$. Afeta milhões de pessoas no mundo. Até $86 \%$ dos pacientes apresentam alterações a nível cervical ${ }^{2}$.

Apesar de acometer todo o eixo vertebral, destaca-se a junção crânio-cervical, devido a presença de articulações sinoviais, sede do envolvimento reumatoide ${ }^{3}$.

Descreve-se ainda uma tenossinovite envolvendo o ligamento transverso e alares, levando a menor resistência à tração e posterior desenvolvimento de subluxação atlantoaxial e impacção atlantoaxial $^{4}$. Estudos mostram esse envolvimento variando de 14-88\%, sendo a terceira localização mais frequente de acometimento pela doença, perdendo apenas para o acometimento de mãos e pés ${ }^{5,6}$.

A literatura revela que acima de $80 \%$ dos pacientes portadores de AR desenvolverão alterações radiográficas da coluna cervical após 10 anos de doença e destes espera-se que em torno de 40\% tenham alterações neurológicas, manifestadas através da deterioração das funções dos membros superiores e inferiores, caracterizando a mielopatia cervical ${ }^{6}$.

O objetivo é reavaliar um grupo de pacientes estudados há 6 anos e avaliar se houve piora do acometimento cervical. Os autores avaliaram o padrão de acometimento cervical a longo prazo em pacientes com diagnóstico de artrite reumatoide, correlacionando os achados de imagem com quadro neurológico, medicação utilizada, tempo de evolução da doença, idade de início da doença, exames laboratoriais e quadro clínico, comparando os resultados.

\section{MATERIAL E MÉTODO}

Estudo de corte transversal. Analisou-se amostra já selecionada previamente. Todos os pacientes com diagnóstico de artrite reumatoide segundo os critérios do American College of Rheumatology (ACR), num total de 24 pacientes.

Os critérios de exclusão foram: cirurgia prévia na coluna cervical, antecedentes de trauma na coluna cervical, início da doença antes dos 18 anos ou abandono do estudo. Os mesmos da série anterior.

Foram avaliados os diversos tipos de instabilidade cervical e os correlacionado com alguns sintomas clínicos (parestesias, cervicalgia, avaliação subjetiva da força e cefaleia occipital). Devido à interferência da atividade da doença, a avaliação objetiva de força foi excluída uma vez que levava a uma dificuldade de interpretação.

O exame físico levou em conta as alterações de reflexos, a presença de clônus e dos sinais de L'Hermitté e Babinsky.

A investigação laboratorial foi realizada através da dosagem da velocidade de hemossedimentação (VHS), proteína $\mathrm{C}$ reativa (PCR) e fator reumatoide (FR).

A análise radiográfica foi realizada através de radiografias simples da coluna cervical nas incidências ântero-posterior, perfil neutro e dinâmico (flexão e extensão), tendo sido padronizado uma distância tubo-filme de $1,5 \mathrm{~m}$.

Além dessas variáveis, também foi avaliado uso e duração da terapia com glicocorticoide, uso de droga anti-reumática modificadora de doença (DARMD), o tempo de doença e a idade ao diagnóstico.

O envolvimento neurológico e a dor foram quantificados através da classificação de $\operatorname{Ranawat}^{7}$ (Tabelas 1 e 2).

Tabela 1. Classificação de Ranawat para dor

\begin{tabular}{c|l}
\hline Classe & \\
\hline 0 & Sem dor \\
\hline$I$ & Dor intermitente, responsiva a analgésicos comuns \\
\hline$I$ & $\begin{array}{l}\text { Dor intermitente, responsiva a analgésicos comuns, necessidade } \\
\text { de imobilização com colar cervical }\end{array}$ \\
\hline III & Dor contínua e incapacitante, não responsivaa analgésicos \\
\hline
\end{tabular}

Adaptado de Ranawat et al?

Tabela 2. Classificação de Ranawat para envolvimento neurológico.

\begin{tabular}{c|l}
\hline Classe & \multicolumn{1}{|c}{ Alteração neurológica } \\
\hline I & Sem anormalidades neurológicas \\
\hline$I I$ & Fraqueza subjetiva, hiper-reflexia, disestesias \\
\hline \multirow{2}{*}{ III } & $\begin{array}{l}\text { Fraqueza objetiva, sinais de trato longo } \\
\text { A - deambulador } \\
\text { B - não deambulador, quadriparético }\end{array}$ \\
\hline
\end{tabular}

Adaptado de Ranawat et al? $^{7}$.

Na avaliação radiográfica foi pesquisada a presença de instabilidade occipito-cervical (invaginação basilar), instabilidade atlanto-axial e instabilidade subaxial.

A invaginação basilar, caracterizada pela migração superior do odontoide, foi avaliada pelos métodos de Ranawat ${ }^{7}$ (valores de referência: maior ou igual a 15 para homens e maior ou igual a 13 para mulheres) (Figura 1) e Redlund-Johnell ${ }^{8}$ (valores de referência: maior ou igual a 34 para homens e 29 para mulheres) (Figura 2). Considerou-se positiva por qualquer um dos critérios.

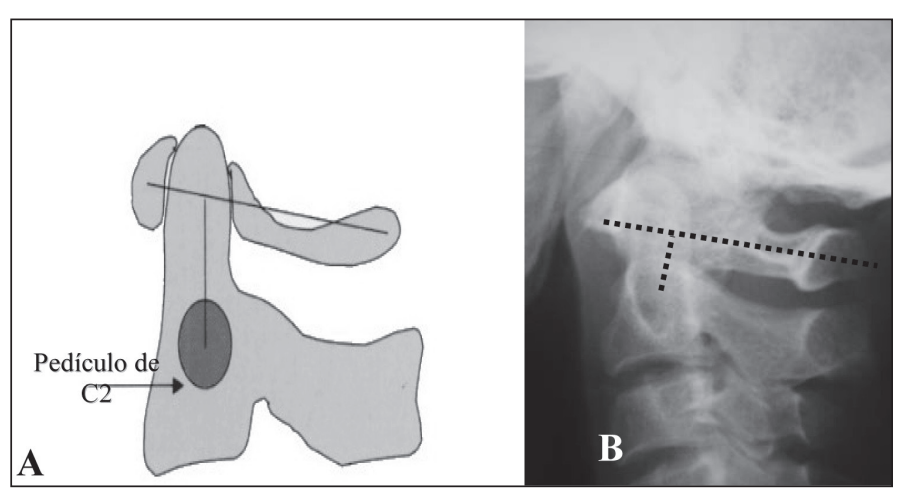

Figura 1. A) Método de Ranawat (medida da distância entre o centro do pedículo de C2 e linha transversa no arco de C1). B) Índice de Ranawat. Radiografia em perfil da coluna cervical. 


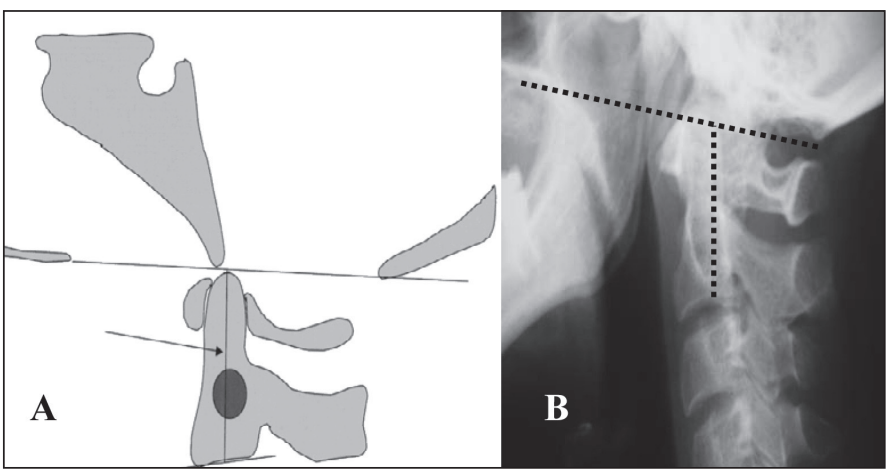

Figura 2. A) Método de Redlund-Johnell (medida da distância entre a linha de McGregor e ponto médio da margem inferior de C2). B) Índice de Redlund-Johnell. Radiografia em perfil da coluna cervical.

A instabilidade atlanto-axial (SAA) foi diagnosticada pela distância atlanto-dental anterior (DADA) maior do que 3mm (medida entre o arco anterior do atlas e o processo odontoide).

A subluxação subaxial (SSA) foi diagnosticada com translação entre uma vértebra e outra maior do que $3,5 \mathrm{~mm}$.

A tabulação dos dados foi feita pelo programa Microsoft ${ }^{\circledR}$ Excel 2007 e a análise estatística pelo SPSS \& (Statistical Package for the Social Sciences) for Windows ${ }^{\circledR}$, versão 15.0. Os testes de regressão logística univariada foram utilizados para avaliar a influência das variáveis no surgimento das instabilidades. Nível de significância: $p<0,05$. Para comparação dos dados do trabalho anterior com os atuais utilizou-se o teste Exato de Fisher.

\section{RESULTADOS}

Dos 35 pacientes estudados anteriormente, 28 foram contatados. Desses, três tinham ido a óbito e um se recusou a participar, totalizando um total de 24 pacientes $(68,5 \%)$. Desses, quatro $(16,6 \%)$ eram do sexo masculino e 20 (83,3\%) do feminino. A média de idade foi de 52,4 anos (variando de 40 a 70 anos).

A idade ao diagnóstico foi, em média, 30,8 anos (variando de 20 a 52 anos) e o tempo em atividade da doença média de 26,9 anos (variando de 8 a 49 anos).

As drogas antireumáticas modificadoras de doença (DARMDs) não eram usadas por oito pacientes (33,3\%). Quatorze pacientes $(58,3 \%)$ faziam uso de apenas uma DARMD, dois $(8,3 \%)$ usavam esquema duplo e nenhum paciente com esquema triplo.

Em relação ao uso de corticoide, dois pacientes não o faziam, sendo que os outros 22 que usavam, faziam-no em média há 20,3 anos (variando de 8 a 49 anos).

Seis pacientes (25\%) que não tiveram sintomatologia ou eram Ranawat 0 (dor), três (12,5\%) apresentavam instabilidades cervicais (um caso de SAA, um caso de SSA e um caso de associação de SAA e SSA).

Sete $(29,1 \%)$ pacientes não apresentavam alterações de reflexos. Um paciente apresentava sinal de Babinsky presente, dois apresentavam o sinal de L'Hermitté positivo e um apresentava clônus.

A velocidade de hemossedimentação (VHS) estava elevada em 14 pacientes (58,3\%), com média de 36,9 mm/h. Dezessete pacientes $(70,8 \%)$ eram fator reumatoide positivo.

Dos pacientes avaliados, 8 (33,3\%) apresentavam a coluna cervical estável. Entre os 16 pacientes $(66,6 \%)$ com quadro radiográfico de instabilidade, nove apresentavam mais de uma instabilidade (cinco casos de SAA + SSA, três casos de associação entre invaginação e SAA e um caso de SAA + SSA + invaginação)

Instabilidade atlanto-axial foi encontrada em 12 pacientes (50\%), sendo que em cinco a DADA era normal na radiografia neutra. A média da DADA na radiografia neutra foi de $4,41 \mathrm{~mm}(1,2$ a 12,1 $\mathrm{mm}$ ) e no perfil em flexão de 7,25 mm (1,2 a 15,2 mm). A mensuração da DADP revelou que um paciente apresentava um intervalo menor que $14 \mathrm{~mm}$ (Figura 4).

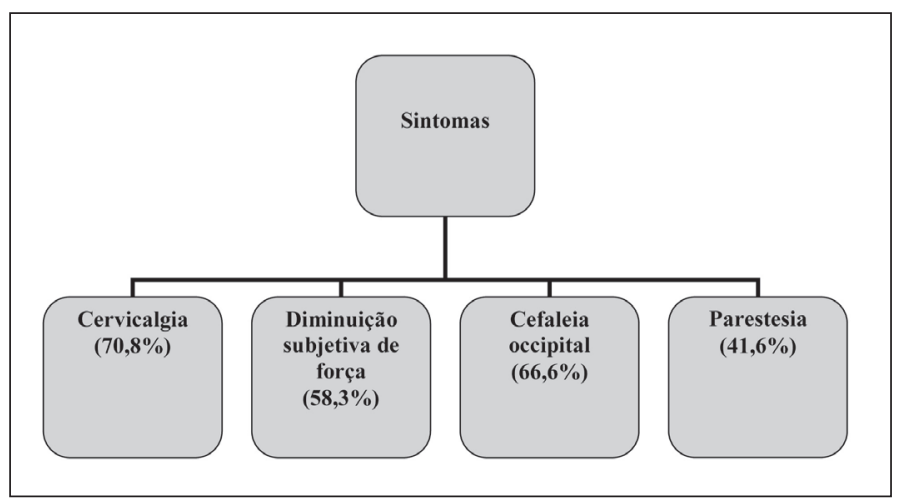

Figura 3. Incidência dos sintomas encontrados na amostra.

Tabela 3. Distribuição de pacientes quanto à classificação de Ranawat (dor).

\begin{tabular}{c|c}
\hline Classe 0 & 5 pacientes $(20,8 \%)$ \\
\hline Classe I & 17 pacientes $(70,8 \%)$ \\
\hline Classe II & 0 pacientes \\
\hline Classe III & 2 pacientes $(8,3 \%)$ \\
\hline
\end{tabular}

Tabela 4. Distribuição de pacientes quanto à classificação de Ranawat (envolvimento neurológico).

\begin{tabular}{c|c}
\hline Classe I & 14 pacientes $(58,3 \%)$ \\
\hline Classe II & 8 pacientes $(33,3 \%)$ \\
\hline Classe III-A & 2 pacientes $(8,3 \%)$ \\
\hline Classe III-B & 0 pacientes \\
\hline
\end{tabular}

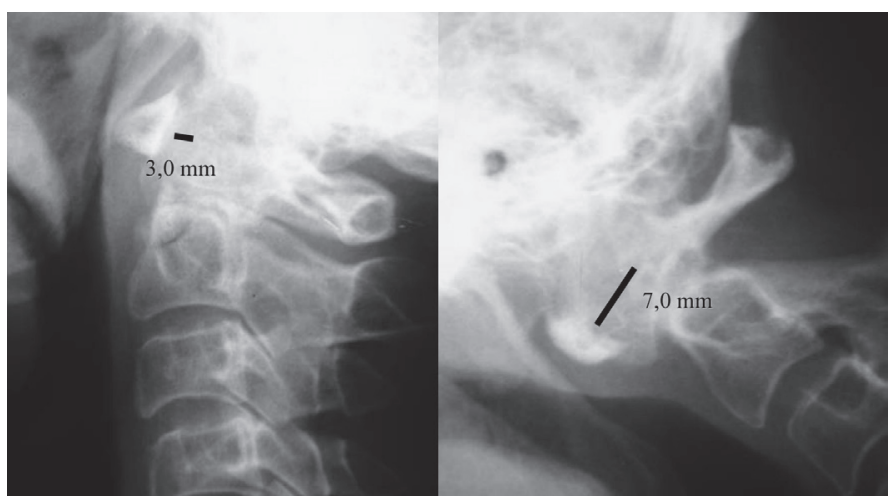

Figura 4. Radiografia em perfil mostrando na incidência em neutro DADA $(3,0 \mathrm{~mm})$ e aumento da DADA no perfil em flexão $(7,0 \mathrm{~mm})$.

A subluxação subaxial foi observada em 10 pacientes $(41,7 \%)$. A invaginação basilar foi encontrada em quatro pacientes $(16,7 \%)$, com diagnóstico pelo método de Ranawat em todos os 4 pacientes, pelo método de Redlund-Johnell em 2 pacientes e diagnóstico simultâneo pelos dois métodos em 2 pacientes (Tabela 5).

A análise estatística demonstrou correlação significativa das seguintes variáveis: reflexo bicciptal $(p=0,049)$ e método de Ranawat ( $p=0,023$, nos raios- $x$ em extensão a $p=0,034$, nos raios- $x$ neutros) com coluna estável, erosão do odontoide $(p=0,048)$ e subluxação lateral $(p=0,022) \quad$ com instabilidade atlanto-axial e sinal de Lhermithé $(p=0,025)$ com invaginação basilar. Não houve correlação significativa das outras variáveis com as instabilidades (subluxação atlanto-axial, invaginação basilar e subluxação subaxial). O teste Exato de Fisher revelou aumento significativamente estatístico da subluxação subaxial, quando comparado com o encontrado há seis anos $(p=0,032)$ (Tabela 6). 
Tabela 5. Distribuição dos pacientes quanto ao tipo de instabilidade cervical.

\begin{tabular}{c|c}
\hline Coluna estável & 8 pacientes $(33,3 \%)$ \\
\hline Instabilidade atlanto-axial & 12 pacientes $(50 \%)$ \\
\hline Instabilidade subaxial & 10 pacientes $(41,6 \%)$ \\
\hline Invaginação basilar & 4 pacientes $(16,6 \%)$ \\
\hline
\end{tabular}

Tabela 6. Números de pacientes de acordo com a instabilidade avaliados em dois momentos.

\begin{tabular}{|c|c|c|c|c|c|}
\hline \multirow{2}{*}{ Fator } & \multicolumn{2}{|c|}{ Antes } & \multicolumn{2}{|c|}{ Após } & \multirow{2}{*}{$\mathbf{p}$} \\
\hline & $n$ & $\%$ & $n$ & $\%$ & \\
\hline \multicolumn{6}{|c|}{ Coluna estável } \\
\hline Não & 22 & 62,9 & 16 & 66,7 & \\
\hline Sim & 13 & 37,1 & 8 & 33,3 & 1,000 \\
\hline Total & 35 & 100,0 & 24 & 100,0 & \\
\hline \multicolumn{6}{|c|}{$\begin{array}{l}\text { Instabilidade } \\
\text { atlanto axial }\end{array}$} \\
\hline Não & 20 & 57,1 & 12 & 50,0 & \\
\hline Sim & 15 & 42,9 & 12 & 50,0 & 0,684 \\
\hline Total & 35 & 100,0 & 24 & 100,0 & \\
\hline \multicolumn{6}{|c|}{$\begin{array}{l}\text { Invaginação } \\
\text { basilar }\end{array}$} \\
\hline Não & 30 & 85,7 & 20 & 83,3 & \\
\hline Sim & 5 & 14,3 & 4 & 16,7 & 1,000 \\
\hline Total & 35 & 100,0 & 24 & 100,0 & \\
\hline \multicolumn{6}{|c|}{$\begin{array}{l}\text { Instabilidade } \\
\text { subaxial }\end{array}$} \\
\hline Não & 27 & 77,1 & 14 & 58,3 & \\
\hline Sim & 8 & 22,9 & 10 & 41,7 & 0,032 \\
\hline Total & 35 & 100,0 & 24 & 100,0 & \\
\hline
\end{tabular}

Teste: Exato de Fisher.

\section{DISCUSSÃO}

Estudos mostram que existe um contraste entre achados clínicos e radiológicos em pacientes portadores de artrite reumatoide, em se tratando de coluna cervical. Entre 10 a 50\% dos pacientes portadores de alterações radiológicas não apresentam qualquer sintoma. Souza e Defino ${ }^{9}$ relatam que não existe correlação estatística significativa entre as instabilidades cervicais e as queixas de cervicalgia ou sinais de compressão neurológica.

Outro importante fator é o aumento da incidência de acometimento cervical com o aumento da idade e tempo em atividade da doença. A média de idade do último trabalho foi de 51,6 e agora encontramos 52,4 anos. O tempo médio de atividade da doença, de 15 anos anteriormente, agora está em 26,9 anos. Ocorreu um aumento geral na incidência das instabilidades: 66,6\%, follow-up de 6 anos, quando comparado ao estudo prévio, que encontrou $62,9 \%{ }^{10}$. A instabilidade atlanto-axial teve aumento percentual de $42,9^{10}$ para $50 \%$ e a invaginação basilar um aumento de $14,3^{10}$ para $16,7 \%$. Mas dentre as instabilidades, destacamos a instabilidade subaxial que teve um aumento de $22,9 \%{ }^{10}$ para $41,7 \%$. Pelo teste Exato de Fisher, observamos que este aumento foi significativamente estatístico, com $p=0,032$. Younes et al destacou um aumento do envolvimento da coluna cervical de $43 \%$ para $70 \%$ em um seguimento longitudinal de pacientes ao longo de 5 anos.

Assim sendo, a avaliação sistemática e periódica da coluna cervical nos pacientes com AR, deveria ser rotina nos serviços de reumatologia e ortopedia. Kwek et al $^{11}$ revela que esta avaliação não é realizada nem como pré-operatório em uma parcela significativa dos pacientes.

O baixo custo e o acesso fácil à radiografia simples a torna o método ideal de triagem das instabilidades da coluna cervical ${ }^{12}$ Entretanto, salienta-se a importância da ressonância magnética quando o paciente apresenta dor cervical incontrolável, alterações neurológicas ou subluxação atlanto-axial à radiografia. Descreve-se uma prevalência de envolvimento cervical de 47,5\% à radiografia simples, 28,2\% à tomografia e 70\% à ressonância magnética.

Assim como no estudo anterior, não encontramos correlação estatística com uso das DARMDs e o desenvolvimento de alterações cervicais

O acometimento cervical em 66,6\% de nossos pacientes encontra-se dentro dos dados da literatura ${ }^{13,14}$ destacando a progressão num seguimento de 6 anos.

O tipo mais frequente de instabilidade cervical em pacientes com A.R é a instabilidade atlanto-axial ${ }^{15}$, o que também foi visto no estudo anterior. Estudos encontraram uma prevalência que varia de $83,6 \%$ a $89,7 \%$ nas séries estudadas. Nossa série encontrou correlações significativamente estatísticas com este tipo de instabilidade: erosão do odontoide $(p=0,048)$ e subluxação lateral $(p=0,022)$.

A mensuração da DADP é importante na investigação da instabilidade cervical $^{15}$. Valores menores que $14 \mathrm{~mm}$ indicam risco de complicações neurológicas. Esta conduta é reforçada pelo estudo de Zeidman e Ducker ${ }^{16}$ que afirmam ser de 13 a 14 mm o espaço mínimo para as estruturas neurais, ao se tratar de junção cranio-cervical. Nossa série encontrou um paciente com DADP menor que $14 \mathrm{~mm}$.

Foi observado em nosso estudo que 41,6\% (5/12) dos pacientes com SAA apresentavam uma DADP normal (menor que $3 \mathrm{~mm}$ ) na radiografia em perfil neutro, também aqui reiterando a importância do estudo dinâmico. Cardoso et al encontraram prevalência de DADA normal na radiografia em perfil neutro de $60 \%$. A diferença entre a taxa de detecção de instabilidades da coluna cervical, levando em conta as radiografias dinâmicas, é estatisticamente significante $(p<0,05)$

O envolvimento da coluna cervical subaxial é considerada a segunda subluxação cervical mais frequente. Conforme a literatura, nossa casuística revelou SSA em 41,6\% dos pacientes. No estudo prévio a porcentagem desse acometimento foi de 22,9\%. Esse expressivo aumento vai ao encontro do que foi visto em estudo realizado $^{16}$, que revela maior acometimento da coluna cervical subaxial em pacientes mais velhos.

Para o diagnóstico da invaginação basilar lança-se mão de diversos métodos, como as linhas de McGregor, Chamberlain e McRae além dos métodos de Redlund-Johnell, Ranawat e Sakaguchi. A literatura traz casos de erosões do dente do áxis além de difícil visualização do palato duro, criando dificuldades para o uso das linhas de McGregor ou McRae. Outro estudo questiona a eficácia do método de Ranawat no diagnóstico das alterações occipito-cervicais, uma vez que só envolve C1-C2, preferindo o método de Redlund-Johnell. Assim sendo, Younes et al defendem o uso de 2 ou 3 métodos para o diagnóstico da instabilidade vertical. De acordo com os autores isso produz uma sensibilidade acima de $90 \%$. Riew et al ${ }^{17}$ complementam através de um estudo, demonstrando que nenhum teste isoladamente tem uma sensibilidade ou valor preditivo negativo maior do que $90 \%$ e sugerem realização de tomografia computadorizada ou ressonância magnética se algum dos testes sugerir invaginação basilar. Optamos em nosso estudo pela utilização dos métodos de Redlund-Johnell e de Ranawat, o que também foi feito na série anterior. Observou-se uma prevalência de invaginação basilar de $16,6 \%$ (quatro pacientes) no nosso grupo de pacientes, com diagnóstico pelo método de Ranawat em quatro pacientes e pelo método de Redlund-Johnell em dois pacientes. O diagnóstico pelos dois métodos foi realizado em dois pacientes. Achado também semelhante ao encontrado no estudo anterior, que foi de 14,3\%. Destacam-se aqui a relevância estatística do método de Ranawat para a confirmação radiológica da coluna 
cervical estável $(p=0,023$ a $p=0,034)$, dado estatístico encontrado em nossa série, além da correlação estatisticamente significativa entre a invaginação basilar e o sinal de Lhermithé $(p=0,025)$. Os outros sinais e sintomas clínicos não tiveram significância estatística na avaliação das instabilidades cervicais

Vesela et al ${ }^{18} \mathrm{em}$ um importante estudo concluem que em geral a intensidade da cervicalgia em pacientes que apresentam alterações no nível C1/C2 diminui com o aumento do intervalo atlanto-dental $(p<0,001)$. Descrevem que esta dinâmica do sintoma dor pode indicar uma intervenção cirúrgica precoce no intuito de se evitar danos maiores, como compressão radicular, mielopatia cervical com progressiva quadriplegia e até mesmo morte súbita. Paus et $\mathrm{a} /{ }^{19}$ indicam cirurgia se o intervalo atlanto-dental for acima de 8-10 mm. Vários trabalhos respaldam uma intervenção cirúrgica mais precoce afim de se reduzir a mortalidade de pacientes portadores de alterações na coluna cervical. Hamilton et al concluem que os resultados cirúrgicos são melhores quando os pacientes encontram-se nas categorias I e II de Ranawat.

Com relação à investigação laboratorial nossa série não encontrou correlação estatística entre as instabilidades e o VHS ou o fator reumatoide positivo, apesar de Paimela et a ${ }^{20}$ descrever 0 fator reumatoide positivo como fator de risco para o acometimento cervical em pacientes portadores de artrite reumatoide.

O aumento da prevalência das instabilidades da coluna cervical nesta série de pacientes previamente avaliados confirma os dados da literatura mundial. Mais uma vez estabelecemos que exista uma frágil associação entre sintomas e achados de imagem. De posse desses dados, reforça-se ainda mais a necessidade de uma investigação rotineira da coluna cervical de pacientes portadores de artrite reumatoide para que se possam prevenir complicações neurológicas graves e até mesmo fatais.

\section{CONCLUSÕES}

A reavaliação dos pacientes após seis anos revelou uma piora do padrão radiográfico da coluna cervical. Houve um aumento absoluto das instabilidades cervicais, com destaque para a instabilidade subaxial, cujo aumento foi significativamente estatístico $(p=0,032)$. Observamos também outras correlações significativamente estatísticas: sinal de Lhermithé e invaginação basilar $(p=0,025)$ além dos achados de erosão do odontoide $(p=0,048)$ e subluxação lateral $(p=0,022)$ com instabilidade atlanto-axial.

\section{REFERÊNCIAS}

1. Ahn JK, Hwang Ji-won, Oh Ji-Min, Lee J, Lee YS; Jeon CH et al. Risk factors for development and progression of atlantoaxial subluxation in Korean pacients with rheumatoid arthritis. Rheumatol Int. 2010;1007:296-1437.

2. Cornejo NM, Villagrán MG, Pucci MP, Ibaceta RS. Columna cervical reumática. Coluna/ Columna. 2009;8(1):94-98.

3. Reiter MF, Boden SD. Inflammatory disorders of the Cervical Spine. Spine. 1998;23(24):2755-66

4. Chen TY, Lin KL, Ho HH. Morphologic Characteristics of Atlantoaxial Complex in Rheumatoid Arthritis and Surgical Consideration Among Chinese. Spine. 2004;29(9):1000-1005.

5. Younes M, Belghali S, Kriâa S, Zrour S, Bejia I et al. Compared imaging of the rheumatoid cervical spine: Prevalence study and associated factors. Joint Bone Spine. 2009;76:361368.

6. Hamilton JD, Johnston A, Madhok HA, Capell R. Factors predictive of subsequente deterioration in rheumatoid cervical myelopathy. Rheumatology. 2001.40:811-815.

7. Ranawat CS, O'Leary P, Pellici P, Tsairis P, Marchisello P, Dorr L. Cervical spine fusion in rheumatoid arthritis. J Bone Joint Surg. 1979;61-A1003-10.

8. Redlund-Johnell I; Pettersson H. Radiografic measurements of the cranio-vertebral region. Acta Radiol Diagn. 1984;25(1):23-8.

9. Souza CP, Defino HLA. Estudo radiográfico das alterações da coluna cervical na artrite reumatoide e sua associação com sinais e sintomas da doença. Acta Ortop Bras. 2005;13(1):38-41.

10. Cardoso ALP, Silva NA, Daher S, Morais FB, Carmo HF. Avaliação da coluna cervical em artrite reumatoide. Rev Bras Ortop. 2010;45(2):160-5

11. KwekTK, Lew TWK, Thoo FL. The role of preoperative cervical spine x-rays in rheumatoid arthritis. Anaesth Intensive Care.1998;26:636-41.

12. Rawlins BA, Girardi FP, Boachie-Adjei O. Rheumatoid arthritis of the cervical spine. Rheum Dis Clin North Am. 1998;24 (1)55-65.

13. Chang DJ; Paget SA. Neurologic complications of rheumatoid arthritis. Rheum Dis Clin North Am. 1993:19(4):955-73.

14. Boden SD. Rheumatoid arthritis of the cervical spine. Surgical decision making based on predictors of paralysis and recovery. Spine. 1994;19(20):2275-80.

15. Maeda T, Saito T, Harimaya K, Shuto T, Iwamoto Y. Atlantoaxial Instability in Neck Retraction and Protusion Positions in Pacients With Rheumatoid Arthritis. Spine. 2004;29(7):757762.

16. Zeidman SM, DuckerTB. Rheumatoid arthritis. Neuroanatomy, compression, and grading of deficts.Spine. 1994;19(20):2259-66.

17. Riew KD, Hilibrand AS, Palumbo MA, Sethi N, Bolhman HH. Diagnosing basilar invagination in the rheumatoid patient: the reliability of radiographic criteria. J Bone Joint Surg Am. 2001;83(2):194-2001

18. Vesela M, Stetkarova I, Lisy J. Prevalence of $\mathrm{C} 1 / \mathrm{C} 2$ involvement in Czech rhuematoid arthritis patients, correlation of pain intensity, and distance of ventral subluxation. Rheumatol Int. 2005;26:12-15

19. Paus AC, Steen H, Roislien J, Mowinckel P, Teigland J. High Mortality Rate in Rheumatoid Arthritis With Subluxation of the Cervical Spine. Spine. 2008;33(21):2278-2283.

20. Paimela L, Laasonen L, Kankaapää E, Leirisalo-Repo, M. Progression of cervical spine changes in patients with early rheumatoid arthritis. J Rheumatol. 1997;24(7):1280-4 